\title{
Eine optimierte automatische Methode zur quantitativen immunologischen Antigen- und Antikörperbestimmung
}

\author{
Von H. Ebeling \\ Aus dem Institut für Klinische Chemie und Klinische Biochemie (Direktor: Prof. Dr. H.-J. Dulce) \\ am Kiinikum Steglitz der Freien Universität Berlin
}

(Eingegangen am 23. Januar/2. März 1973)

Es wird eine standardisierte automatische nephelometrische Methode zur quantitativen Antigen- und Antikörperbestimmung beschrieben. Dazu werden für einige Antigen-Antikörper-Systeme optimale Verhältnisse von Ionenstärke, pH-Wert, Temperatur, Reaktionszeit, Netzmittel-, Antigen- und Antikörper-Konzentration ermittelt.

\section{An optimized automatic metbod for the quantitative immunological determination of antigens and antibodies}

A standardized automatic nephelometric method is described for the quantitative determination of antigens and antibodies. The optimal conditions, i. e. ionic strength, $\mathrm{pH}$, temperature, reaction time, concentrations of wetting agent, antigen and antibody, are reported for some antigen-antibody systems.

Quantitative Angaben einzelner Proteine in Körperflüssigkeiten sind für den Arzt von größerer und spezifischer Aussagekraft als Elektrophoresen mit Gesamteiweißbestimmungen.

Bisher übliche quantitative Methoden, die radiale Immundiffusion und Raketenimmunelektrophorese, sind zeitraubend, teuer und eher semiquantitativ $(1-3)$.

Diese Arbeit lehnt sich an das von Eckman et al. (4) vorgestellte System einer automatischen immunologischen quantitativen Transferrin-Bestimmung an. Anliegen ist es, die Reproduzierbarkeit dieser Bestimmungsweise zu überprüfen und die Methode so zu optimieren und $\mathrm{zu}$ standardisieren, daß sie genau, spezifisch, schnell, preisgünstig und auf andere Proteine, Antigene wie Antikörper, anzuwenden ist.

Mit der Möglichkeit, über diese Methode AntikörperKonzentrationen zu bestimmen, ist ein Weg aufgezeigt, schnell Immunisierungserfolge nach aktiver Schutzimpfung zu kontrollieren.

Quantitative Aussagen über die bei Antigen-Antikörper-Reaktionen sich bildenden Präzipitat-Teilchen kann man mit dem Verfahren nach HeIdelberger (zit. nach KABAT (5)), mit der Turbidimetrie (6) oder, wie auch in der vorliegenden Arbeit, mit der hochempfindlichen Nephelometrie (7) machen. Dabei folgen nephelometrische Konzentrationsbestimmungen einer von RAYLEIG̣H entwickelten Beziehung (zitiert nach (8)):

$$
\mathrm{I}=\mathrm{k} \frac{\mathrm{N} \mathrm{V}}{\mathrm{r}^{2} 1^{4}} \mathrm{I}_{0}
$$

$I=$ Intensität der Streustrablung,

$\mathrm{I}_{0}=$ eingestrahlte Lichtintensität,

$1=$ Wellenlänge des eingestrahlten Lichtes,

$\mathrm{N}=$ Teilchenzahl in der Volumeneinheit,
$\mathrm{V}=$ Teilchenvolumen,

$\mathrm{I}=$ Abstand des Empfängers vom beleuchteten Volumenelement,

$\mathrm{k}=$ Konstante.

Unter konstanten Versuchsbedingungen ( $V, r, 1=$ konstant), niedriger Teilchenkonzentration und geringer Schichtdicke, ist $I / I_{0}$ proportional der Teilchenkonzentration.

Ein Hauptproblem, das Konstanthalten der PräzipitatTeilchengröße, setzt Einhalten konstanter Reaktionsbedingungen voraus. Um eine möglichst hohe Streustrahlungsintensität $\mathrm{zu}$ erhalten, werden optimale Ionenstärken, $\mathrm{pH}$-Werte, Temperaturen, Realtionszeiten, Netzmittelzusätze, Antigen- und AntikörperKonzentrationen in dieser Arbeit ermittelt.

\section{Material und Methoden}

Apparate

Technicon Auto Analyzer:

Sampler II

Proportioning Pump

Manifold

Transformer und Volstat

II Fluoronephelometer

Flowcell 013-B008-01.

Recorder und Chart No R0483

Pumpenschläuche

Primär- und Sekundärfilter von G. K. Turner Association 110 bis 812, narrow pass, (entsprechend $436 \mathrm{~nm}$ ).

Photometer Eppendorf, $\mathrm{Hg}$-Filter $546 \mathrm{~nm}$.

Spektralphotometer PMQ II von C. Zeiss.

\section{Reagenzien}

Monospezifische Antiscra gegen Human-Plasmaproteine vom Kaninchen (Bchringwerke $A G$ ),

Standard-Human-Scrum, stabilisiert (Bchringwerke $A G$ ),

Human-Albumin, trocken, reinst (Behringwerke AG),

Human-Transferrin, rcinst (Behringwrerke AG), 
Cocruloplasmin-Standard, lyophil getrocknet (Behringwerke AG), Fibrinogen (Hyland, Q-PAK TM-Coagulation F. Reference Plasma, Human, dried),

Partigen Immundiffusionsplatten (Behringwerke AG).

Britton-Robinson Puffer (9)-NaCl-Lösung: mit tridest. Wasser:

Lösung I:

$2,85 \mathrm{mmol} / 1$ Citronensäure nach Sörensen (Merck),

2,85 mmol/1 Monokaliumdihydrogenphosphat p. a. (Merck),

$2,85 \mathrm{mmol} / \mathrm{l} \mathrm{Barbital}$ p. a. (Merck),

2,85 mmol/1 Borsäure nach SörENSEN (Merck),

$61,6 \mathrm{mmol} / 1 \mathrm{NaCl}$

Lösung II:

$20 \mathrm{mmol} / \mathrm{l}$ Natriumhydroxid-Plätzchen p. a. (Merck),

$61,6 \mathrm{mmol} / 1 \mathrm{NaCl}$.

Mit $100 \mathrm{ml}$ Lösung I plus $38,3 \mathrm{ml}$ Lösung II wird auf $\mathrm{pH}$ 6,00, unter Kontrolle mit der pH-Glaselektrode, eingestellt. Die fertige Lösung hat eine Gesamtsalzkonzentration von $75,4 \mathrm{mmol} / \mathrm{l}$.

Triton-Wasser: 1 Tropfen $(=35 \mathrm{mg}$ ) Wetting Agent Triton $\mathrm{x}-100$ (Technicon Chemicals S. A.) ad 11 tridest. Wasser.

\section{Zur Eisenbestimmung}

Merckotest Eisen Reagenziensatz (Merck).

Zur Bestimmung der totalen Eisenbindungskapazität außerdem

0,44 mol/l Natriumsulfat, wasserfrei, p. a. (Merck),

$0,59 \mathrm{~mol} / \mathrm{l}$ Magnesiumsulfat p. a. (Merck),

Eisen-III-Lösung $\vdots 0,179 \mathrm{mmol} / 1 \mathrm{FeCl}_{3} \cdot 6 \mathrm{H}_{2} \mathrm{O}, 10 \mathrm{mmol} / 1 \mathrm{HCl}$.

Zur Proteinbestimmung bei $215 / 225 \mathrm{~nm}$

$1,58 \mathrm{~mol} / 1 \mathrm{Natriumsulfat}$, wasserfrei, p. a. (Merck),

Diäthyläther p. a. (Merck).

\section{Zur zupeidimensionalen Geldiffusion}

Agarose, reinst, (Serva),

Veronal-Puffer:

$0,07 \mathrm{~mol} / 1$ Barbital p. a. (Merck),

$1,38 \mathrm{~mol} / 1$ 1,3-Propandiol ( $96-98 \%$ GC, Schuchardt) werden mit etwa $0,06 \mathrm{~mol} / 1 \mathrm{NaOH}$ auf $\mathrm{pH} 8,6$ eingestellt.

Agarose-Gel-Lösung:

$15 \mathrm{~g}$ Agarose werden ad 11 gelöst mit einem Teil Veronal-Puffer und drei Teilen tridest. Wasser.

\section{Vorgehen}

Im Prinzip werden alle Versuche nach dem Fließschema (Abb. 1) durchgeführt. Durch das kontinuierliche Hindurchfließen der Reaktionsgemische durch die Meßküvette soll ein Aggregieren und Sedimentieren der Präzipitatteilchen verhindert werden. Bei

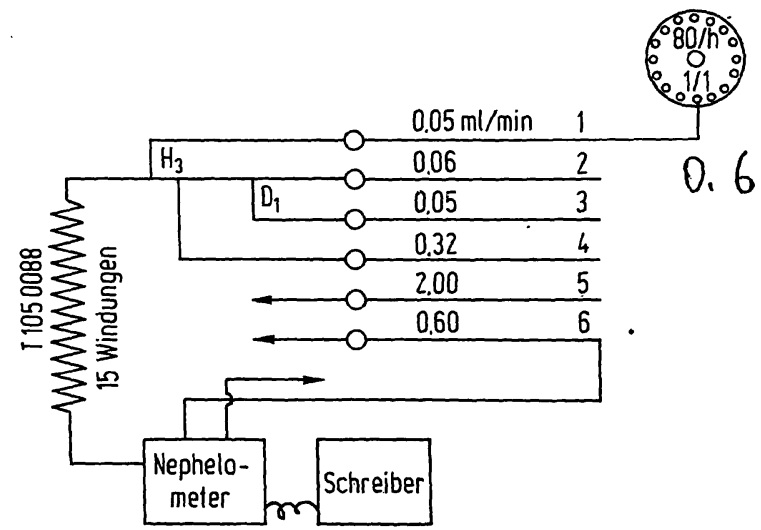

Abb. 1. Fließschema

a) zur Antigenbestimmung: Schlauch Nr. 1 = Antigenprobe; Nr. $2=$ Triton-Wasser; Nr. 3 = Antikörper-Lösung; Nr. 4 = Luft; $\circ \mathrm{Nr} .5=$ Waschflüssigkeit zum Probennehmer (Lösung wie bei Nr. 2); Nr. $6=$
Küvettenabsaugung
b) zur Antikörperbestimmung: Schlauch Nr. $1=$ Antikörperprobe; Nr. 3 = Antigenlösung; sonst wie bei a) allen Versuchen wird streng auf visuell partikelfreie Lösungen vor Gebrauch geachtet. Zeigen sich Partikel, so werden die Lösungen durch entstaubte Papierfaltenfilter in staubfreie Gefäße filtriert. Nach den jeweiligen Versuchen wird das Durchlaufsystem gründlich mit tridest. Wasser gespült. Der Probennehmer- und Antikörper-Schlauch (Schlauch Nr. 1 und 3) werden zuvor durch etwa $15 \mathrm{~min}$ langes Ansaugen von $0,1 \mathrm{~mol} / \mathrm{l} \mathrm{NaOH}$ von Proteinresten befreit.

Antigen- wie Antikörper-Lösuingen, soweit es sich um Plasma oder Serum handelt, haben eine vom Verdünnungsgrad abhängige Eigenstreuung, die stets mitberücksichtigt werden muß $(3,4)$.

I. Bei der Antigenbestimmung wird die Eigenstreuung der AntiseraLösungen als Basislinie durch einen etwa 10 - bis 15 minütigen Antikörpervorlauf eingestellt. Dabei läßt man den Probenschlauch (Nr. 1) nur Probenverdünnungsmittel und Schlauch Nr. 2 TritonWasser ansaugen. Verläuft die Basislinie parallel zum linken Schreiberrand, werden die Proben über Schlauch Nr. 1 angesaugt. Die Basis kann mit dem Baseline-Knopf am Fluoronephelometer auf eine gewünschte Höhe eingestellt werden. Beim Ansaugen der Proben empfiehlt es sich, mit der höchsten Eichwert-Konzentration anzufangen. Diese Konzentration muß in der "Antikörperüberschuß"-Zone der HEIDELBERGER-Kurve (Abb. 8) liegen $(5,10)$. In einem Vorversuch wird durch Verstellen des Standard Calibration-Knopfes am Fluoronephelometer eine zweckmäßige Spreizung dieses Meßpeaks erhalten.

Die Eigenstreuung der Proben wird ermittelt, indem der Antikörperschlauch Nr. 3 nur das. Antikörperverdünnungsmittel ohne Antiserum ansaugt. Gegen diese Basis bestimmt man die Eigenstreuung der Proben. Es muß dabei auf gleiche Apparat-Verstärkung wie bei der Antigen-Antikörper-Reaktion geachtet werden.

Bei der Auswertung ergibt sich die wahre Streulichtintensität des Antigen-Antikörper-Komplexes aus der Differenz Gesamtpeakhöhe minus Eigenstreuungspeakhöhe (Abb. 2).

II. Bei der Antikörperbestimmung wird ähnlich wie bei der Anțgenbestimmung verfahren. Die Basis wird durch ständiges Ansaugen von Antigen-Lösung über den Schlauch Nr. 3 eingestellt. Sie entspricht dann der Eigenstreuung dieser Lösung. Die Proben sind die verschiedenen Antikörper-Konzentrationen. Als

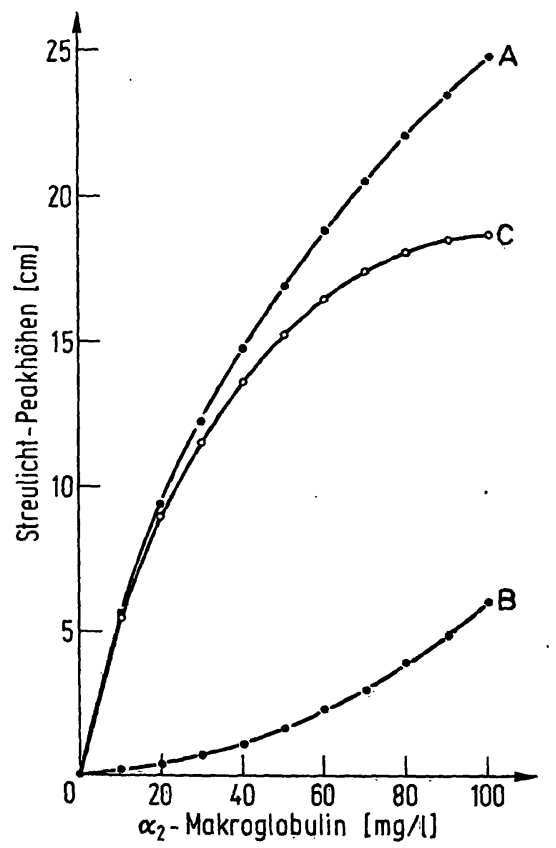

Abb. 2 Auswerten bei Berücksichtigen von Eigenstreuung, $\alpha_{2}-$ Makroglobulin
in Standard-Human-Serum Charge 570 . Antikörper-(1:6 vorverdünnt) in Standard-Human-Serum Charge 570. Antikörper- (1:6 vorverdünnt) und Antigen-Lösungen in BRIT: ON-ROBINSON-Puffer mit 0,038 mol/1 $\mathrm{NaCl}, \mathrm{pH}$
der Proben; Kurve $\mathrm{B}=\mathrm{A}=$ Immunpräzipitat-plus Eigen-Streuung der Proben; Kurve $B=$ Probeneigenstreuung; Kurve $C=$ Differenzplexstreuungs-Kurve 
Eichlösung dient cine Lösung mit bekannter Antikörper-Konzentration. Von ihr stellt man eine Verdünnungsreihe her, die man als Probe über Schlauch Nr. 1 ansaugen läßt. Die Eichwerte müssen auf dem aufsteigenden Schenkel, der sog. „Antigenüberschuß"-Zone bei Antikörperbestimmungen liegen (Abb. 9).

Auch hicrbei muß die Eigenstreuung der Antikörperproben ermittelt werden. Man läßt dazu über Schlauch Nr. 3 das Antigenverdünnungsmittel ansaugen und bestimmt gegen diese Basis durch Ansaugen der Antikörper-Proben über Schlauch Nr. 1 die Eigenstreuung der Antikörper-Lösungen.

Die Bestimmungsweise der reinen Antigen-Antikörper-Streulichtintensität ist die gleiche wie bei der Antigen-Methode (s. o.).

III. Antikörper-Konzentrationsbestimmung der Antikörper-Ausgangslösung (modifiziert nach Averdunk et al. (11)):

1. Åquivalenzpunkts-Bestimmung über den Automaten, Vorgehen wie bei Antigenbestimmung.

2. Herstellen einer Lösung mit der Antigen-Konzentration des Äquivalenzpunktes.

3. Herstellen folgender Lösungen durch den Automaten:

Lösung A: Schlauch Nr. 1 und 3 in Puffer, Nr. 2 in Triton-Wasser. Lösung B: Nr. 2 und 3 wie bei A, Nr. 1 in Antigen-Lösung.

Lösung C: Nr. 1 und 2 wie bei $A, N r .3$ in Antikörper-Lösung. Lösung D: Nr. 1 wie bei B, Nr. 2 wie bei $A, N r .3$ wie bei $C$.

Etwa $5 \mathrm{ml}$ dieser Lösungen werden jeweils durch Unterbrechen des Durchlaufsystems nach der Mischschlange aufgefangen und über Nacht stehengelassen. Von Lösung $D$ wird nach Zentrifugieren der Uberstand verwendet. Aus $B$ und $C$ und dem Uberstand von $D$ werden gegen Lösung $A$ ( = Leerwert) die Proteingehalte durch Messen der Extinktionen bei $278 \mathrm{~nm}$ am Zeiss-Spektralphotometer an einer mit Reinst-Human-Albumin hergestellten Eichkurve bestimmt.

Der Proteingehalt der Antikörper-Ausgangslösung errechnet sich folgendermaßen:

Die Eiweißgehalte der Lösungen $B$ und $C$ werden addiert. Davon wird der Proteingehalt des Uberstandes von Lösung $D$ subtrahiert. Es ergibt sich der Proteingehalt des abzentrifugierten Antigen-Antikörper-Präzipitates. Durch Subtraktion der bekannten Antigen-Eiweißkonzentration am Äquivalenzpunkt vom Proteingehalt des Präzipitates erhält man durch Multiplikation mit dem Antikörper-Vorverdünnungsfaktor die AntikörperProteinkonzentration der Ausgangslösung.

Die Äquivalenzpunktsbedingungen werden zusätzlich überprüft. Dazu wird der Uberstand der Lösung D durch zweidimensionale Doppeldiffusionstechnik auf Vorhandensein von Antikörpern und Antigenen hin untersucht $(5,11,12)$.

\section{Ergebnisse und Diskussion}

In der Literatur $(3,4,11,13-15)$ werden fast ausnahmslos standardisierte Reaktionsgemische mit Salzkonzentrationen um oder höher als $0,15 \mathrm{~mol} / \mathrm{l}$ angegeben. Dabei wird ebenso wenig wie beim $\mathrm{pH}$-Wert auf optimale Verhältnisse geachtet. Optimale Immunpräzipitate erhält man, wẹnn sowohl die Antigen- als auch die Antikörper-Proben mit einer Puffer-SalzLösung von $75,4 \mathrm{mmol} / 1$ und $\mathrm{pH} 6,00$ vorverdünnt werden (Abb. 3 u. 4). Im Reaktionsgemisch herrscht dann, infolge der Weiteryerdünnung über den Automaten, eine Puffer-Salz-Konzentration von $11 \mathrm{mmol} / \mathrm{l}$. Bei höheren Salzkonzentrationen kommt es bei Zwischenspülen mit Tritonwasser zu Nachpeaks und somit zu schlechter Trennschärfe. Die Ursache dafür ist, $\mathrm{da} \beta$ man durch Zwischenspülen mit salzfreier Lösung optimalere Bedingungen bezüglich der Salzkonzentration schafft. So kommt es während des Aus-

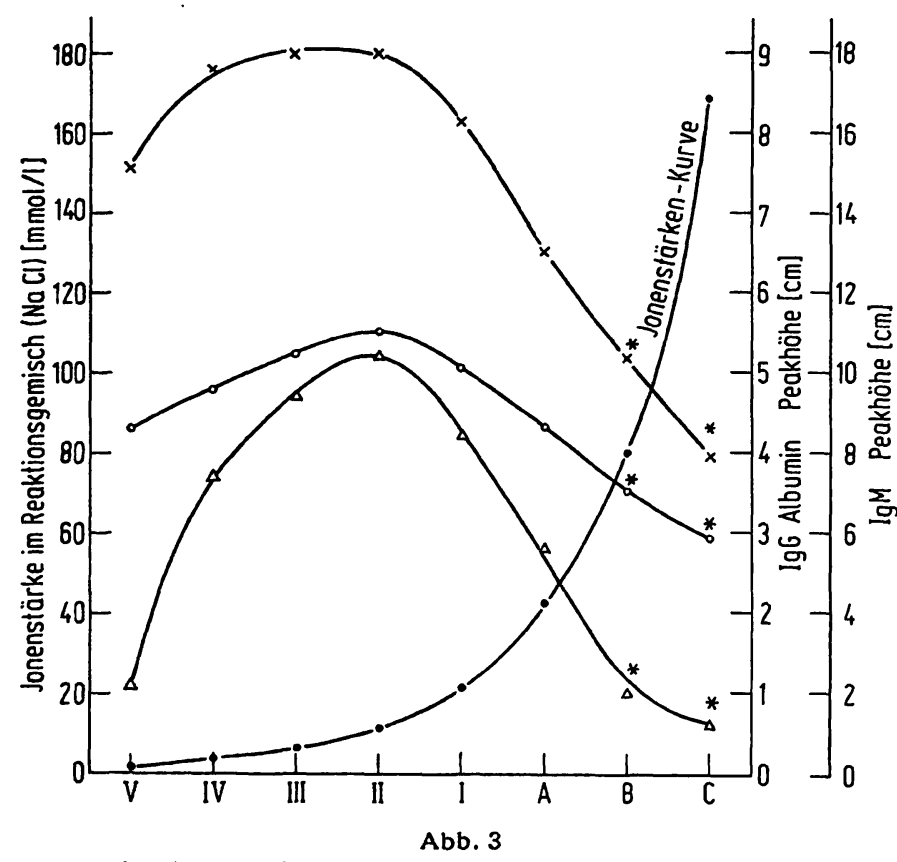

Abhängigkeit der Meßpeakhöhe von der Ionenstärke ( -0 ). Abszisse : Ansätze der Antigen- $(\angle-\triangle \mathrm{Ig} \mathrm{G}=9,45 \mathrm{mg} / \mathrm{l}, \times-\times \mathrm{Ig} M=8,0$
$\mathrm{mg} / \mathrm{l}$, $\mathrm{O}-\mathrm{O}$ - Albumin $=26,027 \mathrm{mg} / \mathrm{l}$ Standard-Human-Serum) und Antikörper-Lösungen ( $1: 6$ vorverdünnt) mit NaCl-Lösungen in mol/1: $V=0,0$, IV $=0,0192, I I I=0,0385, I I=0,077, \quad I=0,154, A=$
$0,3078, B$ 0,3078, $\mathrm{B}=0,615, \mathrm{C}=1,23$. Ordinate - links: Ionenstärke der

höhen in $\mathrm{cm}$ (verstärkungs- und antigenkonzentrationsabhängig).

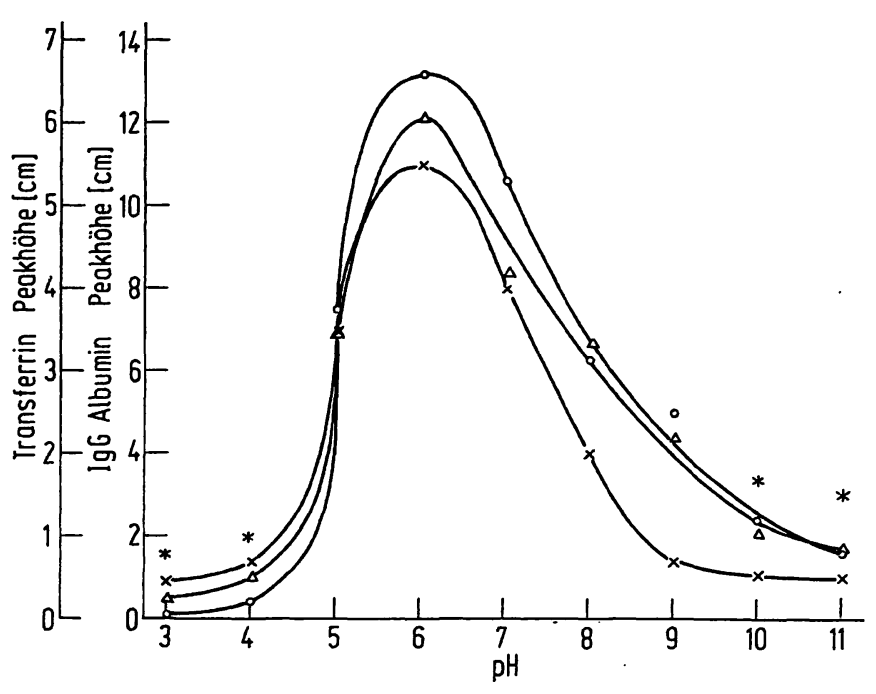

Abb. 4

Abhängigkeit der Meßpeakhöhe vom pH-Wert: Abszisse: pH-Werte. Ansätze der Antigen- $(O-O$ Albumin $=26,027 \mathrm{mg} / 1, \times-\times 1 \mathrm{Ig}=$
$9,09 \mathrm{mg} / \mathrm{l}$ aus Standard-Human-Serum Charge $570 ; \Delta-\triangle$ ReinstTransferrin $=40 \mathrm{mg} / \mathrm{l}$ ) und Antikörperlösungen (jeweils $1: 6$ vorverdünnt) in BRITTON-ROBINSON-Puffer- $\mathrm{NaCl}-(0,0385 \mathrm{~mol} / \mathrm{I} \mathrm{NaCl})$ Lösung des jeweiligen pH-Wertes. Ordinate: Relative Peakhöhen in cm (ver-
stärkungs- und antigenkonzentrationsabhängig). * Schlechte Trennschärfe der Peaks

waschens zur weiteren Antigen-Antikörper-Komplexbildung, die sich in Nachpeaks bemerkbar macht.

Als optimale Reaktionstemperatur erweist sich Raumtemperatur $\left(23,5^{\circ} \mathrm{C}\right.$ ) (Abb. 5). Die reine Reaktionszeitdauer soll etwa $31 / 2 \mathrm{~min}$ betragen, um optimale Streulichtintensitäten zu messen (Abb. 6). Bei l. c. $(4,11,15)$ werden höhere Temperaturen und bei l. c. $(3,4,11,15)$ längere Reaktionszeiten neben den besprochenen anderen Milieubedingungen beschrieben. Zusetzen von etwa $30 \mathrm{mg}$ Netzmittel Triton $x-100 \mathrm{zu}$ einem Liter Reaktionsgemisch führt zu optimalen 


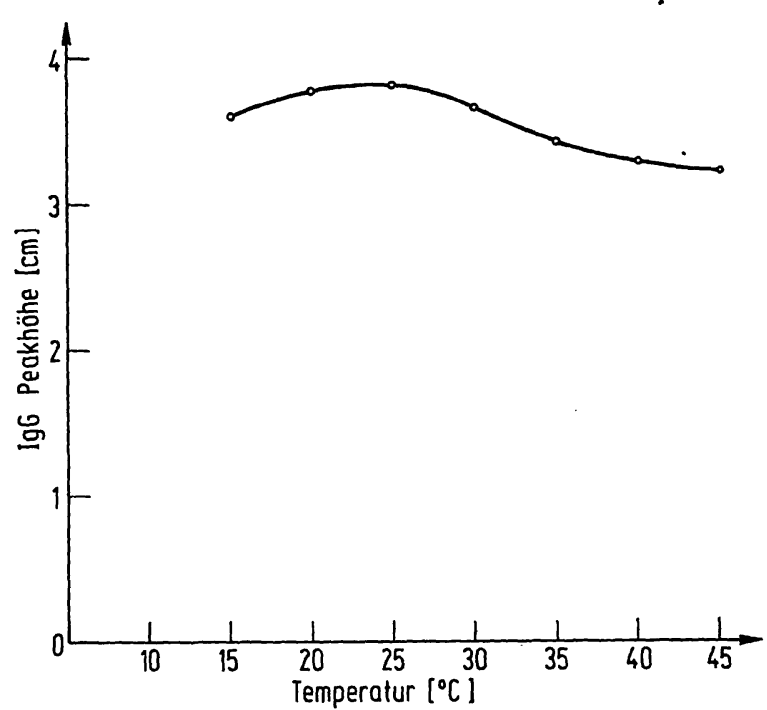

Abb. 5

Antigen-Antikörper-Komplexbildung in Abhängigkeit von der Temperatur: Abszisse: Temperatur in ${ }^{\circ} \mathrm{C}$. Die Glasmischschlange ist bei diesen Versuchen durch ein mit der jeweiligen Temperatur thermostatisiertes Wasserbad geleitet. Ordinate: Relative Peakhöhen in $\mathrm{cm}$ als Ausdruck der Antigen-Antikörper-Komplexbildung (verstärkungsund konzentrationsabhängig). Ig $G$ aus Standard-Human-Serum $9,45 \mathrm{mg} / \mathrm{l}$. Antigen- wie Antikörper-Lösung (1:6 vorverdünnt) jeweils mit BRITTON-ROBINSON-Puffer pH 6,00 in $83 \mathrm{mmol} / \mathrm{l} \mathrm{NaCl}$ angesetzt

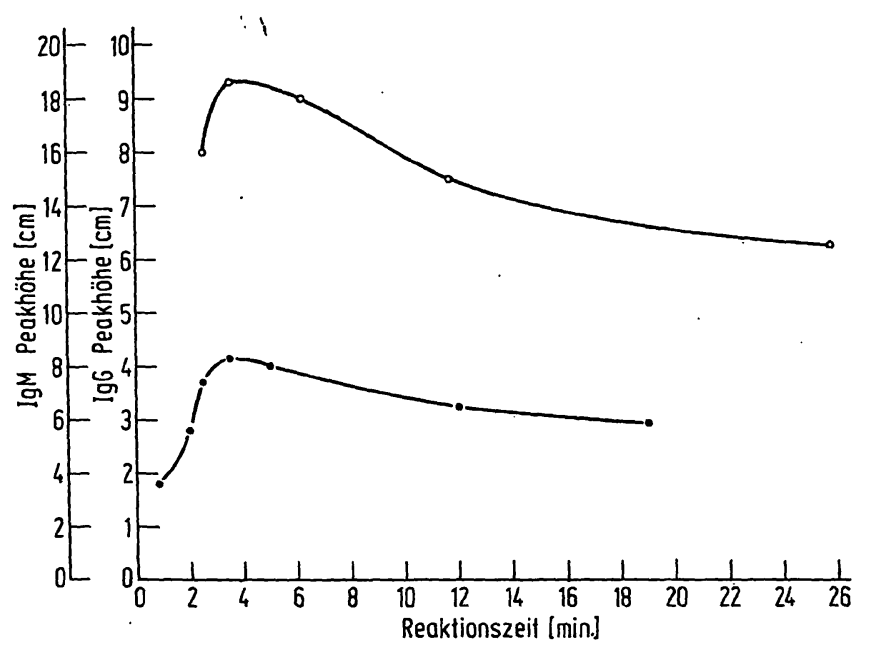

Abb. 6

Antigen-Antikörper-Komplexbildung in Abhängigkeit von der Reaktionsdauer: Abszisse: Reaktionszeit in Minuten. Die verschieden langen Inkubationszeiten werden mit dazwischengeschalteten unterschiedlich langen Glasschlangen erzielt. Ordinate: Komplexbildung ausgedrück in $\mathrm{cm}$ Peakhöhe (verstärkungs- und konzentrationsabhängig). 0-6 Ig G $=9,45 \mathrm{mg} / \mathrm{l}, O-O \mathrm{Ig} \mathrm{M}=10 \mathrm{mg} / \mathrm{l}$ aus Standard-Human-Serum und Antikörper-Lösungen (1:6 vorverdünnt) in BRITTON-ROBINSON Puffer mit $75 \mathrm{mmol} / \mathrm{l} \mathrm{NaCl}$, pH 6,00 bzw. mit $38 \mathrm{mmol} / \mathrm{l} \mathrm{NaCl}, \mathrm{pH} 5,5$

Peakhöhen im Gegensatz zu in der Literatur angegebenen größeren Mengen (4) (Abb. 7). Ein Weglassen von Netzmittel führt wegen einer von uns häufiger beobachteten Instabilität der Antisera-Lösungen beim Durchlaufen des Systems zu Ausfällungen.

Aufgrund der an einigen Beispielen untersuchten optimalen Verhältnisse von Antigen-Antikörper-Reaktionen empfiehlt sich folgendes standardisiertes Vorgehen. Beim Bestimmen von Präalbumin, Albumin, saurem $\alpha_{1}$-Glykoprotein, $\alpha_{1}$-Antitrypsin, Coeruloplasmin, $\alpha_{2}$-Makroglobulin, $\beta 1 C$-Globulin, Transferrin, Fibrinogen, Immunglobulin $G, A$ und $M$ aus menschlichen Körperflüssigkeiten hat sich ein 1:6-Vorverdünnen der benutzten monospezifischen Antisera mit

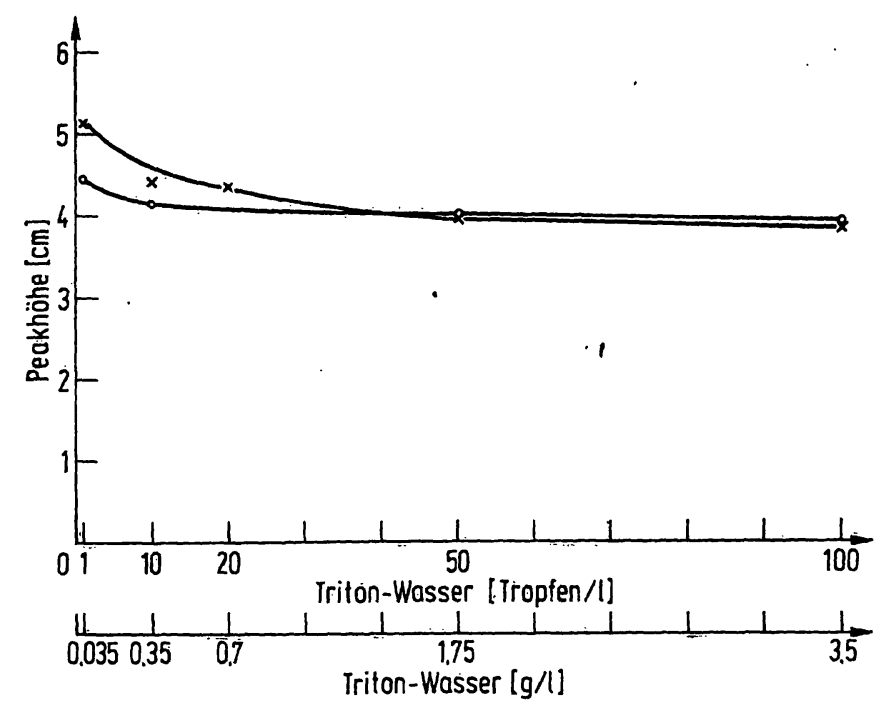

Abb. 7

Antigen-Antikörper-Komplexbildung, ausgedrückt in der Peakhöhe, in Abhängigkeit von der Netzmittelkonzentration (Triton $x-100$ ): Abszisse: Triton $x-100-M e n g e$ in Tropfenzahl bzw. $g$ ad 11 tridest. Wasser $(: 1,16=$ Triton $x-100-$ Konzentration $/ 11$ Reaktionsgemisch). Ordinate: Relative Peakhöhen in $\mathrm{cm}$ (verstärkungs- und kon-

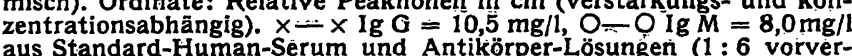
aus Standard-Human-Serum und Antikorper-Lósungen (1:6 vorver$38,5 \mathrm{mmol} / \mathrm{I} \mathrm{NaCl}, \mathrm{pH} 5,5$. Weglassen von Netzmittel führt häufig $\mathrm{zu}$ Ausfallen von Antikörpern

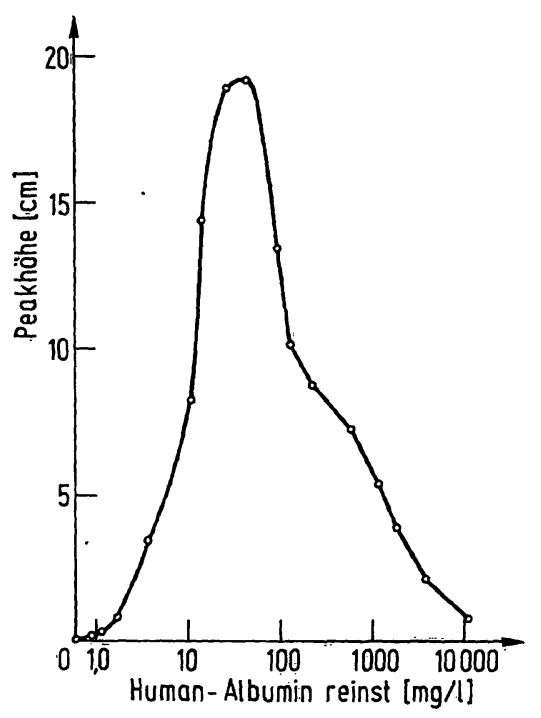

Abb. 8

Abhängigkeit der Antigen-Antikörperkomplexbildung von der Antigenkonzentration (= "HEIDELBERGER-Kurve"): Abszisse: Antigen-Konzentration ( = Albumin/reinst) in mg/l. Ordinate: Peakhöhe in cm. Antialbumin-(1:6 vorverdünnt) und Antigen-Lösung inBRITTON-ROBINSONPuffer, pH 6,00 mit 61,5 mmol/1 NaCl. Linker aufsteigender Schenkel $=$ "Antikörperüberschuß"-Zone, Wendepunktsbereich $=$ Äquivalenzzone, rechter absteigender Schenkel = ,Antigenüberschuß"-Zone

75,4 mmol/1 Britton-RoBinson-Puffer-NaCl-Lösung, $\mathrm{pH}$ 6,00, bewährt. Als Antigen-Eichstandard wird ein Standard-Human-Serum, ein Coeruloplasmin-bzw. Fibrinogen-Standard benutzt. Die Antigen-Lösungen werden ebenfalls mit der erprobten Puffer-Salz-Lösung vorverdünnt. Für die beschriebene Antikörper-Vorverdünnung werden Antigen-Vorverdünnungen im Konzentrationsbereich von 3,0 bis $50,0 \mathrm{mg} / \mathrm{l}$ hergestellt. Nur der steil aufsteigende linke Schenkel der HeIdelberger-Kurve ( = „Antikörperüberschuß“Zone) kommt dabei als Meßbereich in Frage (Abb. 8). Die zu untersuchende unbekannte Probe wird so vor- 
verdünnt, daß sie bei normal hoher Konzentration im mittleren Teil des Meßbereichs der Eichkurve läge. $\mathrm{Da}$ eine hohe Antigenkonzentration leicht zu einem Antigenüberschuß und somit $\mathrm{zu}$ einer fehlerhaften Bestimmung führen könnte, sollte man aus Sicherheitsgründen stets eine zweite Konzentration - und zwar eine 1:2 Verdünnung der Vorverdünnung - mitbestimmen. Dabei muß die Probe mit der höheren Konzentration auch einen größeren Peak, die mit der höheren Verdünnung einen kleineren Peak ergeben. Sind die Peaks etwa gleich groß, befinden sich die Konzentrationen um den Äquivalenzpunktsbereich. Ist der Peak mit der kleineren Konzentration größer als der mit der geringeren Verdünnung, so befinden sich die Konzentrationen auf dem absteigenden rechten Schenkel der Heidelberger-Kurve. In beiden Fällen muß die Probe stark vorverdünnt werden, um sie in den gültigen Konzentrationsbereich der Eichkurve zu bringen.

Für Antigenkonzentrationen kleiner als $3,0 \mathrm{mg} / \mathrm{l} \mathrm{muß}$ die Antikörperkonzentration weiter verringert werden, um optimale Antigen-Antikörper-Verhältnisse zu erhalten. Wie aus Abbildung 9 hervorgeht, lösen Antikörperkonzentrationen, die höher sind als die an den Gipfelpunkten dieser Kurven, die Antigen-AntikörperKomplexe wieder auf. So muß man, um AntigenKonzentrationen kleiner als $6,0 \mathrm{mg} / 1$ optimal zu bestimmen, die benutzte Antikörperausgangslösung um mehr als $1: 4$ vorverdünnen. Bei Antigen-Konzentrationen kleiner als $3,0 \mathrm{mg} / \mathrm{l} \mathrm{mu}$ die Antikörper-Lösung um mehr als 1:8 vorverdünnt werden, und bei Antigen-Konzentrationen kleiner als $0,6 \mathrm{mg} / 1 \mathrm{mu}$ die Antikörper-Vorverdünnung mehr als $1: 40$ betragen (Abb. 9).

Aus Abbildung 9 geht außerdem hervor, daß bei konstanter Antigen-Konzentration der aufsteigende

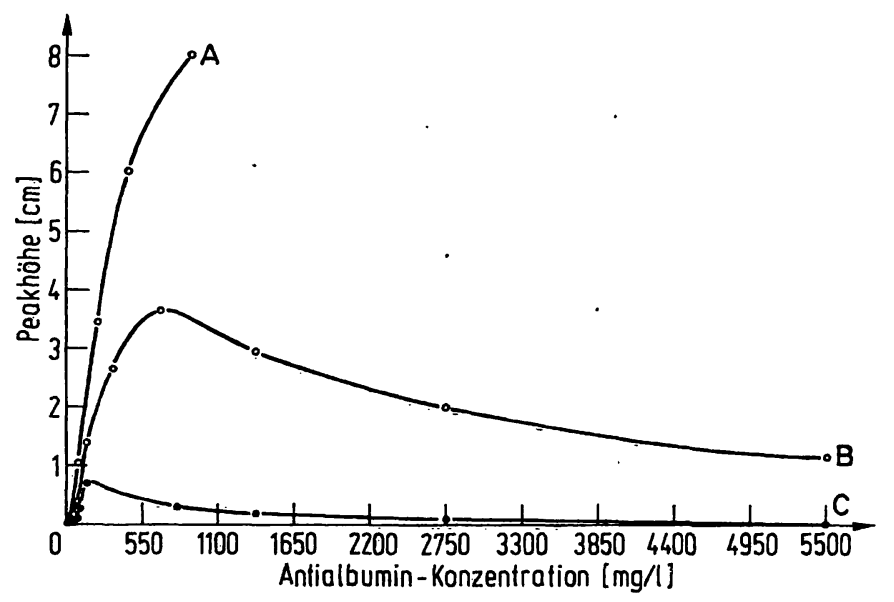

Abb. 9

Abhängigkeit der Antigen-Antikörper-Komplexbildung, dargestellt als Peakhöhen, von uer Antikörperkonzentration: Abszisse: Antialbumin-Konzentration in $\mathrm{mg} / \mathrm{l}$ (Ausgangslösung $5500 \mathrm{mg} / \mathrm{l}$ ). Ordinate: Relative Peakhöhen in $\mathrm{cm}$ (antigenkonzentrations- und verstärkungsabhängig). Antigen-Humanalbumin reinst $(A=6,322 \mathrm{mg} / \mathrm{l}, \mathrm{B}=$ $3,161 \mathrm{mg} / 1$, $C=0,652 \mathrm{mg} / 1)$ und die Antikörper-Verdünnungen in BRITTON-ROBINSON-Puffer pH 6,00, Gesamt-Puffer-Salz-Konzentration 75,4 mmol/l. Linker aufsteigender Schenkel = Antigenüberschu $B^{11}$ Zone, Wendepunktsbereich $\Rightarrow$ optimale" Antigen-Antikörperverhăltnisse, rechter absteigender Schenkel $=$ "Antihörper-Öberschuß"-

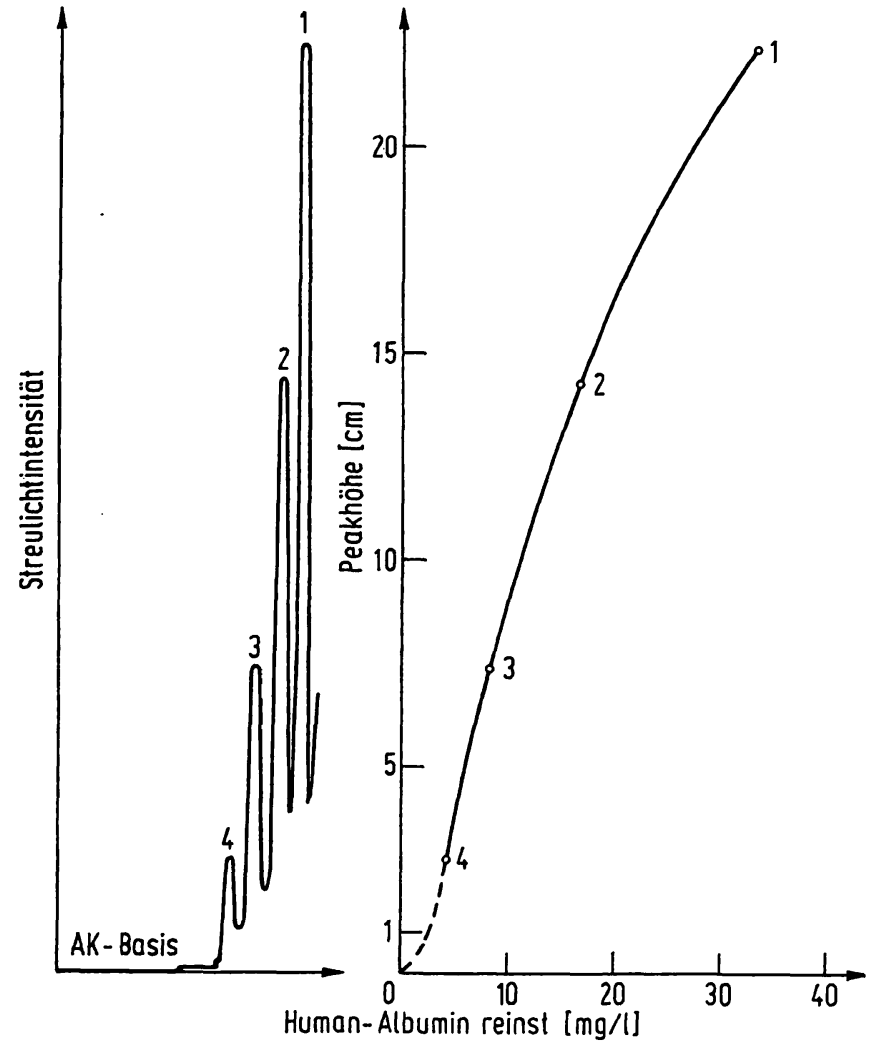

Abb. 10

links: Streulichtintensitäts-Peaks mit vier verschiederıen Albuminreinst-Eichlösungen $(1=33,413 \mathrm{mg} / \mathrm{l}, 2=16,706 \mathrm{mg} / \mathrm{l}, 3=8,353 \mathrm{mg} / 1$; $4=4,176 \mathrm{mg} / \mathrm{l})$, Bestimmungsrate 80 Proben/Stunde $(1: 1)$, Antialbumin- (1: 6) vorverdünnt) Lösung und Antigene in BRITTONrechts: Graphische Auswertung der Eichpeaks: Abszisse: HumanAlbumin-reinst-Konzentration in mg/l. Ordinate: Peakhöhe in $\mathrm{cm}$ die Peaks den reinen Antigen-Antikörper-Präzipitat-Teilchen

linke Schenkel dieser Kurven (die sog. „Antigenüberschuß“-Zone dieser Antikörperkurven) zur AntikörperKonzentrationsbestimmung benutzt werden kann. Sinngemäß gilt auch zur Antikörperbestimmung das bei der Antigenbestimmung bezüglich Eichen und Vorverdünnen der Proben Gesagte.

Die automatische Konzentrationsbestimmung von Antigen oder Antikörper dauert vom Ansaugen der Probe bis zum Schreiberausschlag $280 \mathrm{~s}$. Bei dem geschilderten optimierten und standardisierten Vorgehen befindet sich die Reaktion im steady state, sichtbar an sauberen Peakformen mit waagerechtem Plateau. Diese Optimierung läßt eine Bestimmungsrate von 80 Proben pro Stunde zu. Dabei wird im zeitlichen Verhältnis von 1:1 Probe angesaugt und zwischengespült (Abb. 10). Die Präzision dieser Methode in der Serie und von Tag zu Tag ist am Immunglobulin $G$ überprüft worden.

1. Präzision in der Serie für drei verschiedene Konzentrationen:

a) $10 \mathrm{mg} / \mathrm{l}, \mathrm{n}=26, \overrightarrow{\mathrm{x}} \pm \mathrm{s}=9,85 \pm 0,17 \mathrm{~cm}$, $\mathrm{VK}=1,73 \%$

b) $20 \mathrm{mg} / 1, \mathrm{n}=27, \overline{\mathrm{x}} \pm \mathrm{s}=17,46 \pm 0,24 \mathrm{~cm}$, $\mathrm{VK}=1,37 \%$

c) $30 \mathrm{mg} / \mathrm{l}, \mathrm{n}=24, \overline{\mathrm{x}} \pm \mathrm{s}=21,38 \pm 0,27 \mathrm{~cm}$, $\mathrm{VK}=1,27 \%$. 
2. Präzision von Tag zu Tag:

$\mathrm{n}=20$ Tage, Immunglobulin G-Konzentration

$\overline{\mathrm{x}} \pm \mathrm{s}=11,5 \pm 0,233 \mathrm{mg} / \mathrm{l}, \mathrm{VK}=2,03 \%$.

Der Präzisionsvergleich in der Serie am Beispiel Trans-

ferrin ergibt für die radiale Immundiffusion mit der Partigenplatte einen Variationskoeffizienten von 10 bis $20 \%$ im Gegensatz zu dieser Methode mit 1,5\%.

Es wird am Beispiel Albumin und Transferrin die Richtigkeit der Konzentrationsangabe im Standard-
Human-Serum überprüft. Bei Bestimmen des Albumins im Standard-Human-Serum nach (16) bei 215 und $225 \mathrm{~nm}$ ergibt sich fast $100 \%$ Übereinstimmung mit dem Albumin-Sollwert. Ein Bestimmen der totalen Eisenbindungskapazität (modifiziert nach 1. c. (17)) ergibt gleichfalls eine $100 \%$ Utbereinstimmung mit der Sollwertangabe, wenn ein Transferrin-Mol.-Gew. von 90000 und Vollsättigung pro .Molekül mit zwei Eisen[III]-Atomen zugrunde gelegt 'wird.

\section{Literatur}

1. Mancini, G., Carbonara, A. O. \& Heremans, J. F. (1963), Immunochemistry 2, 235-254. - 2. LAURELL, C.-B. (1966), Biochemistry 15, 45-52. - 3. Larson, Ch., Orenstein, P. \& Ritchie, R. F. (1970), Technicon International Congress, Vol. 1, Clinical, 9-12; (1972), Technicon Auto-Analyzer II, Clin. Method No 12/Preliminary, 1-5. - 4. Eckman, I., Robbins, J. B., VAN Den Hamer, C. J. A., Lentz, J. \& Scheinberg, I. H. (1970), Clin. Chem. 16, 558-561. - 5. KaBAT, E. A (1971), Einführung in die Immunchemie und Immunologie, S. $53 \mathrm{ff}$., Springer-Verlag, Berlin-Heidelberg-New York. - 6. SchwICK, H. G. (1965), Immunchemie, S. 55-67, Springer-Verlag, Berlin-HeidelbergNew York. - 7. Gitlin, D. \& EDELHOCH, H. (1951), J. Immunol. 66, 67-77. - 8. Franke, R. \& ThIELE, K. (1969), Physikalischchemische Methoden im klinischen Laboratorium, Bd. II, S.
97-105, VEB Verlag Volk und Gesundheit, Berlin. - 9. Documenta GeIGY (1968), Wissenschaftliche Tabellen, 7. Aufl. S. $274 \mathrm{ff}$. 10. Heidelber GER, M. (1965), Immunchemie, S. 6-11, SpringerVerlag, Berlin-Heidelberg-New York. - 11. AvERDUNK, R. \& Busse, V. (1969), diese Z. 3, 287-290. - 12. OuchrerLoNY, Ö. (1965), Immunchemie, S. 13-35, Springer-Verlag, BerlinHeidelberg-New York. - 13. Killingsworth, L. M. \& SAvORY, J. (1971), Clin. Chem. 17, 936-940. - 14. SAvory, J., HeintGes, M. G., Killingsworth, L. M. \& PotTer, J. M. (1972), Clin. Chem. 18, 37-42. - 15. Kilingesworth, L. M., Savory, J. \& Teague, P. O. (1971), Clin. Chem. 17, 374-377. - 16. Waddel, W. J. (1956), J. Lab. Clin. Med. 48, 311-314. - 17. FürrR, J. \& Stary, E. (1970), Technicon Symposium, Sonderdruck R 874.

\section{Dr. Herwig Ebeling}

Institut für Klinische Chemie und Klinische Biochemie am Klinikum Steglitz der Frẹien Universität Berlin 1 Berlin 45

Hindenburgdamm 30 